Sir,

\title{
Diffraction by an absorbing wedge
}

At the recent C.N.R.S. conference at Marseille "Protection acoustique des zones d'habitation par desecrans" Pierce [1] gave a paper on the diffraction by an absorbing wedge. The incident wave being an oblique plane wave. The wedge faces were assumed to have an impedance boundary condition of the form

$$
\frac{\partial p}{\partial n}=i k \beta p, \quad \operatorname{Re}(\beta)>0,
$$

where the sound wave has time harmonic variation $e^{-i w t}$ and $k=w / c$, $c$ is the velocity of sound, $n$ the normal pointing into the wedge faces, and $\beta$ the complex specific admittance of the acoustic material comprising the wedge. Although the solution to the impedance wedge problem was solved sometime ago for plane wave incidence, Williams [2], Malyzhinets [3], little use has been made of the solutions because of the extremely complicated functions involved in the solutions, Pierce [1], was trying to somehow make the solution more amenable to practical situations. In particular he was interested in extending the solution to allow for a point source. The line source solution has in fact already been given, Rawlins [4]. One very important future application Pierce [1] was interested in was the application to more complicated barrier structures which involve more than one wedge, i.e. the thick absorbing barrier or the trapezoidal absorbing barrier. By using the diffraction coefficient for the absorbing wedge in conjunction with Kellers method of geometric diffraction [5], any number of barrier shapes could be handled.

The object of the present letter is to suggest a much simpler approach to the absorbing wedge. A recent paper by Butler [6] showed 
that the experimental results of Maekawa [7] on absorbing barriers agreed well with the theoretical results for a perfectly absorbing half plane. The theoretical solution for a perfectly absorbing half plane is obtained by adding the theoretical solution for the hard and soft half plane together and dividing by two. Naturally the same incident wave is used in the hard and soft solution. A simple extension of this technique can be applied to the perfectly absorbing wedge. The solution for all important types of incident sound source are known for the hard and soft wedge, Bowman, Senior \& Uslenghi [8], chapter 6 . Thus by simple superposition the absorbing wedge problem (for any sound source) is easily obtained and the functions required in the calculation of the field are vastly simpler than those used by Pierce [1]. For example the solution for the absorbing wedge with a point sound source is given by adding the expressions (6.106) p.270 and (6.129) p.272 of [8] together and dividing by two. Thus the field for an absorbing wedge is given by

$$
\text { Vabs. }=\frac{1}{2 \pi i \nu} \int_{c_{1}+c_{2}} \frac{e^{i k R(\alpha)}}{k R(\alpha)} \cot \left(\frac{\pi-\alpha-\phi-\phi_{0}}{2 \nu}\right) d \alpha
$$

where the incident wave is

$$
\frac{e^{i k R}}{k R}
$$

and where the various quantities are given by (see fig. 1 and 2),

$$
\begin{aligned}
& R=V\left\{\rho^{2}+\rho_{0}^{2}-2 \rho \rho_{0} \cos \left(\phi-\phi_{0}\right)+\left(z-z_{0}\right)^{2}\right\}, \\
& R(\alpha)=\sqrt{ }\left\{\rho^{2}+\rho_{0}^{2}+2 \rho \rho_{0} \cos \alpha+\left(z-z_{0}\right)^{2}\right\}, \quad \nu=\frac{2(\pi-\Omega)}{\pi} .
\end{aligned}
$$

Various asymptotic techniques can now be applied to (1) to obtain the near or for field. However, this is unnecessary since all the 
asymptotic results are already given for the hard and soft wedge in [8]. An expression for the near field is obtained from [8] by adding the expression (6.108) and (6.131) and dividing the result by two. This gives

$$
\text { Vabs. }=\frac{1}{\nu} \sum_{n=0}^{\infty} \varepsilon_{n} \cos \frac{n\left(\phi-\phi_{0}\right)}{\nu} s_{n / \nu},
$$

where $\varepsilon_{\mathrm{n}}=1$ for $\mathrm{n}=0$ and $\varepsilon_{\mathrm{n}}=2$ for $\mathrm{n}=1,2,3, \ldots$,

$$
s_{i}=\frac{i}{2 k} \int_{-\infty}^{\infty} e^{i t\left(z-z_{o}\right)} J_{\tau}\left(\rho<\sqrt{ }\left(k^{2}-t^{2}\right)\right) H_{\tau}^{(1)}\left(\rho>\sqrt{ }\left(k^{2}-t^{2}\right)\right) d t .
$$

Various asymptotic representations of $\mathrm{S}_{\tau}$ are given in [8]. In particular if $k \rho<<1$ then $(6.112)$ and $(6.135)$ of [8] give

$$
\begin{gathered}
\text { Vabs. }=\frac{i}{\nu} h_{0}{ }^{(1)}\left(k \sqrt{ } /\left\{\rho^{2}+\rho_{0}^{2}+\left(z-z_{0}\right)^{2}\right\}\right) \\
+\frac{2 i}{\Gamma(1 / \nu)}\left(\frac{1}{2} k \rho_{0}\right)^{\frac{1}{v}} \cdot \frac{h_{1 / \nu}^{(1)}\left(k \sqrt{ }\left\{\rho^{2}+\rho_{0}^{2}+\left(z-z_{0}\right)^{2}\right\}\right)}{\left(\sqrt{ }\left\{\rho^{2}+\rho_{0}^{2}+\left(z-z_{0}\right)^{2}\right\}\right)^{1 / \nu}} \cos \frac{\left(\phi-\phi_{0}\right)}{\nu} \\
+o\left[(k \rho)^{\min (2 / \nu, 2)}\right],
\end{gathered}
$$

where $\mathrm{f}_{1 / \nu}^{(1)}$ is the spherical Hankel function, [8] p.58.

The expression for the far field $k \rho \rho_{0} / R_{1}>>1$ is given by (6.113) $(6.114),(6.117)(6.118)$ and $(6.136),(6.137),(6.140),(6.141)$ from [8] as

$$
\text { Vabs. }=\sum_{n 1} \frac{\exp \left\{i k R\left(\alpha_{n 1}\right)\right\}}{k R\left(\alpha_{n 1}\right)}+\left\{\operatorname{Vd}\left(-\pi-\phi+\phi_{0}\right)-\operatorname{Vd}\left(\pi-\phi+\phi_{0}\right)\right\},
$$

where

$$
\begin{aligned}
& \operatorname{Vd}(\beta)=\frac{1}{2 \pi \nu} \int_{0}^{\infty} \frac{e^{i k R(i t)}}{\operatorname{kR}(i t)} \cdot \frac{\sin (\beta / \nu)}{\operatorname{ch}(t / \nu)-\cos (\beta / \nu)} d t, \\
& R_{1}=\sqrt{ }\left\{\left(\rho+\rho_{0}\right)^{2}+\left(z-z_{0}\right)^{2}\right\}, \quad \alpha_{n 1}=\pi-\phi+\phi_{0}-2_{n} 1 \pi \nu \text {, and the summation } \\
& \text { extends over al1 integers } n 1 \text { satisfying the inequality }\left|\phi-\phi_{0}+2 n 1 \nu \pi\right|<\pi .
\end{aligned}
$$


Various asymptotic expressions are given for $\mathrm{Vd}(\beta)$ in [8]. An expression for the diffracted far field for an absorbing wedge is given by adding $(6.124)$ and $(6.144)$ of $[8]$ and dividing by two. Thus

$$
\underset{\operatorname{dibs}}{\operatorname{diff}}=\frac{e^{i\left(k R_{1}+\pi / 4\right)}}{\sqrt{\left(2 \pi k R_{1}\right)}} \cdot \frac{1}{k \sqrt{\rho \rho_{0}}} \cdot \frac{1}{\nu} \sin \frac{\pi}{\nu} /\left(\cos \frac{\pi}{\nu}-\cos \frac{(\phi-\phi 0)}{\nu}\right) .
$$

It can be seen that the technique outlined above is simple and the expressions obtained are not as complex as those obtained in [1], [2], [3]. The above theoretical estimates will give an upper bound to the attenuation expected for practical absorbing wedges. However, the simplicity of the theoretical results combined an empirical approach will probabiy givegood design criteria for Engineers. It is worth mentioning that the same technique can be used to obtain the field scattered by perfectly absorbing bodies of other geometries i.e. strip, ellipse, cone, etc. The hard and soft solutions to many of the important shapes occurring in diffraction are given in [8], for various sources.

\section{$\underline{\text { References }}$}

(1) A.D. Pierce. Theory of sound diffraction around absorbing barrier. Conference C.N.R.S. Marseille 1975.

(2) W.E. Williams. Diffraction of an E-polarised plane wave by an imperfectly conducting wedge. Proc. Roy. Soc. Lon. A252 (1959) p. 367.

(3) G.D. Malyuzhinets. Excitation, reflection and emission of surface waves from a wedge with given face impedances. Soviet Phys Docklady 3 (1958) p.752.

(4) A.D. Rawlins. First Science Research Council Fellow Report. The University of Dundee, 1972.

(5) J.B. Keller. Geometric theory of diffraction. J. Opt.Soc. Am. 52 (1962) p.116.

(6) G.F. Butler. A note on improving the attenuation given by a noise barrier. J. Sound Vib. 32 (1974) p.367.

(7) Z Maekawa. Noise reduction by screens. Memoirs of Faculty of Engineering, Kobe University No. 11, 29 (1965).

(8) J.J. Bowman, T.B.A. Senior, P.L.E. Uslenghi. Electromagnetic and acoustic scattering by simple shapes. North Holland Publishing Co., Amsterdam. 1969. 


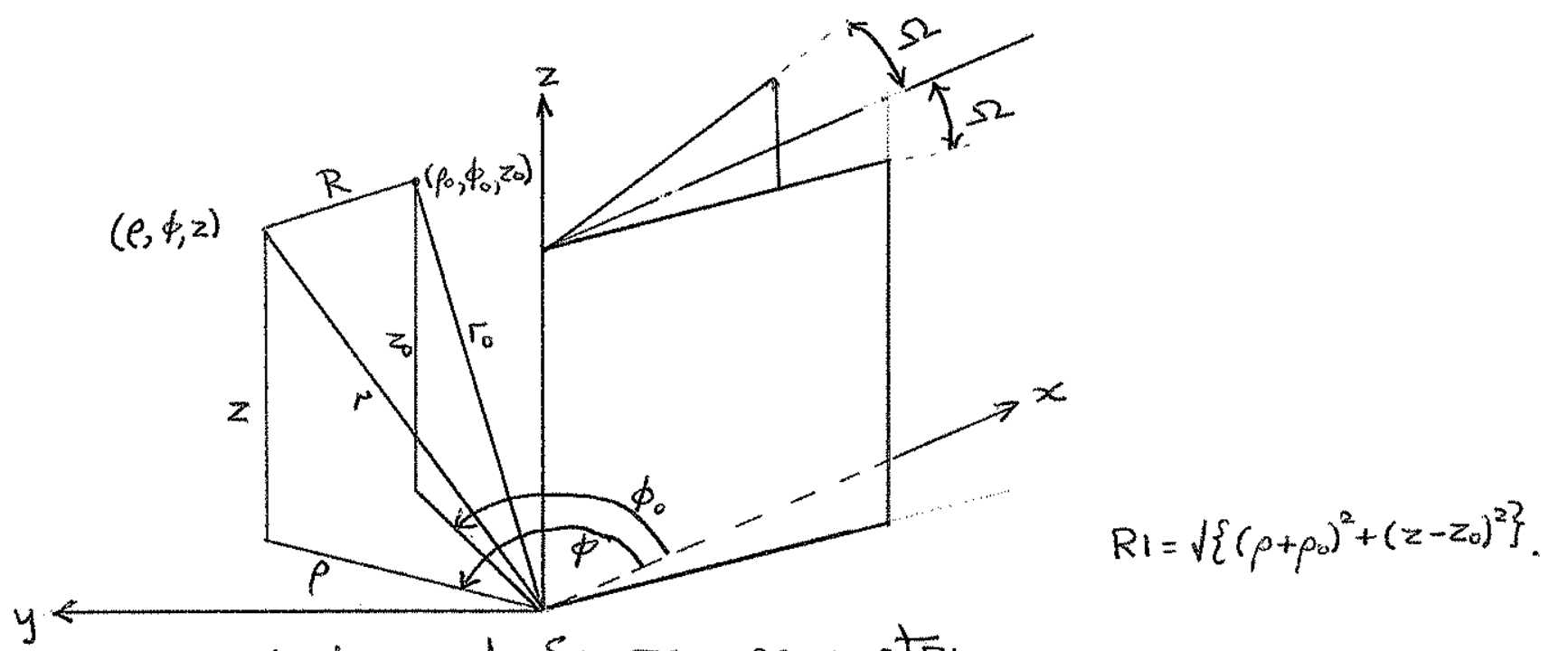

Wedge and Source geometry. figure l.

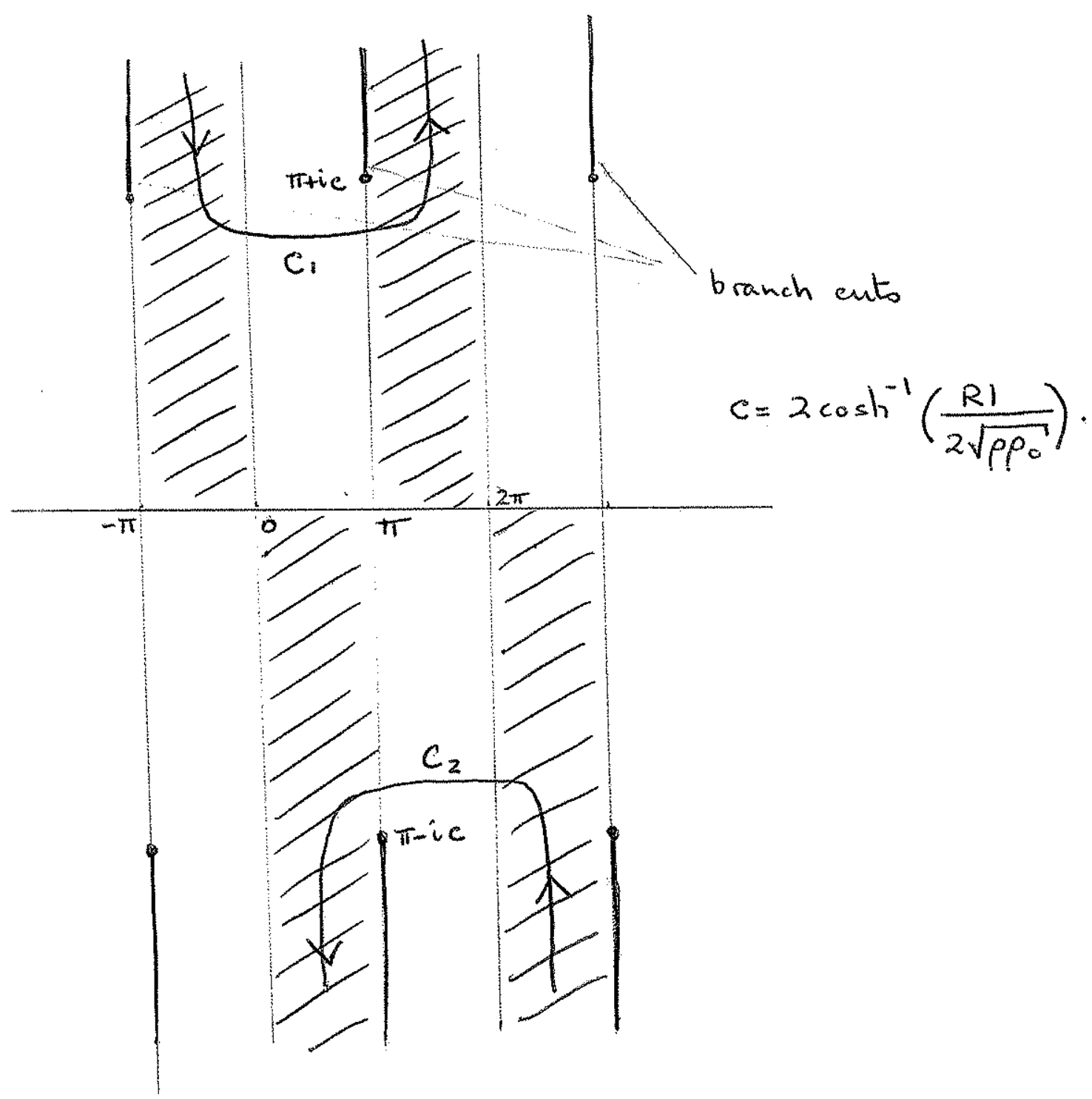

Contours of integration $c_{1}+c_{2}$. figure 2. 
Dear Sir,

When suggesting the simple technique for dealing with an absorbing wedge Rawlins [1] the work of Raman and Krishnan [2] was known to me. However one major drawback of using their technique is that the solution violates the reciprocity condition concerning the interchangeability of the source and receiver, see Rschevkins [3] p. 360. Butlex's [4] model also has this defect. The method might however agree, within a limited range, with experimental work. It is interesting to note that a similar technique to [2], [4] has been suggested by Johasson [5]. However this solution also violates the reciprocity condition, see Pierce [6].

I shal1 now comment on the point raised about curved surfaces. By adding the hard and soft solution together the primary reflected wave (that is, the reflected wave according to geometrical acoustics) from the illuminated portion of any curved surface will vanish. This follows from the fact that at any point of the soft/hard scatterer we can construct a soft/hard tangent plane and apply the normal laws of reflection from a plane. We will take as a specific example the sphere, Bowman et al [7], chapter 10. The high frequency reflected wave for a soft sphere is given by expression (10.35) of [7] and for a hard sphere by expression (10.98). It can be seen that on adding these two terms together the primary reflected wave vanishes. The terms which remain are due to the curvature of the scatterer. These are at least of order $(\mathrm{ka})^{-1}$, where $k$ is the wave number and ' $a$ ' the radius of the sphere, and, since $\mathrm{ka}$ is large, are of second order effect compared to the primary reflected wave. It is possible that a more efficient model than [1] can be introduced to absorb these wave terms due to curvature. At this stage I would not like to hazard a guess as to what it is. A possible approach would be to maximise the expression for the power absorbed by a curved body. In the absence of any other model I suggest that [1] is a suitable approximate model for perfectly absorbing curved surfaces even though the total reflected wave does not vanish. 
[1] A.D. Rawlins. Diffraction by an absorbing wedge.

J. Sound \& Vib. (1975) 41 (3) p. 391-393.

[2] C.V. Raman \& K.S. Krishnan. The diffraction of light by metallic screens. Proc. Roy. Soc. A 116 (1927) p. 254-267.

[3] S.N. Rschevkin. The theory of sound. Pergamon Press 1963.

[4] G.F. Butler. Diffraction by a wedge with an impedance condition at the surface. Jour. Sound \& Vib.

[5] H.G. Jonasson. Diffraction by wedges of finite acoustic impedance with applications to depressed roads.

J. Sound \& Vib. (1972) 25 (4) pp. 577-585.

[6] A.D. Pierce. 1975 Conference C.N.R.S. Marseille 1975.

Theory of sound diffraction around absorbing barriers.

[7] J.J. Bowman, T.B.A. Senior \& P.L.E. Usenght. Electromagnetic and acoustic scattering by simple shapes. Amsterdam : North Holland Publishing Company 1969. 